\title{
Transurethral Needle Electrode Resection of Bladder Tumor: A Technique Obtaining En Bloc Resection and Obviating Obturator Nerve Stimulation
}

\author{
Hongwei Yanga, c, Lixin Shi ${ }^{\mathrm{b}, \mathrm{c}}$, Guangfu Chen ${ }^{\mathrm{b}}$, Weijun Fu ${ }^{\mathrm{b}}$, Jiangping Gao ${ }^{\mathrm{b}}$, \\ Shengkun Sun ${ }^{\mathrm{b}, \mathrm{d}}$, Axiang $\mathrm{Xu}^{\mathrm{b}}$,, Xu Zhang ${ }^{\mathrm{b}}$
}

\begin{abstract}
Background: Transurethral resection of bladder tumor (TURBT) is the current gold standard for treatment of non-muscle-invasive bladder cancer. However, obturator nerve reflex (ONR) remains as a threat during treating lateral wall tumors. The present study aimed to describe a simple and reliable method which provides en bloc resection without ONR.

Methods: Forty-six patients planned for TURBT with superficial bladder carcinoma nested in lateral bladder wall received transurethral needle electrode resection of bladder tumor (TUNER-BT) under epidural anesthesia without obturator nerve block. Intraoperative and postoperative complications were observed. The resected tissues were examined by a pathologist who recorded grade, invasion of the muscularis propria and the presence of muscular invasion.
\end{abstract}

Results: Tumors were multiple in $11(24 \%)$ patients and single in 35 $(76 \%)$ patients. Mean tumor size was $1.79 \pm 0.43 \mathrm{~cm}$ with a mean resection time of $10.8 \pm 4.8$ minutes. None of the 46 patients developed obturator jerks or perforation. Precise histological evaluation was achieved in all cases: $32.5 \%$ pTa, $56.4 \%$ pT1, and $11.1 \%$ pT2.

Conclusions: The needle electrode can be used safely and conveniently during transurethral electroresection of bladder tumors located in the lateral bladder wall. This technique is easy to perform and allows en bloc resection of tumors. The ONR privilege makes TUNER$\mathrm{BT}$ a preferable option when treating tumors located in lateral bladder wall.

Keywords: Bladder cancer; Needle electrode; Obturator nerve block; Obturator nerve reflex; Transurethral resection of bladder tumor

Manuscript accepted for publication August 18, 2015

aDepartment of Urology, Shenyang PLA General Hospital, Shenyang, China bDepartment of Urology, Chinese PLA General Hospital, Beijing, China

${ }^{\mathrm{c}}$ These authors contributed equally.

${ }^{\mathrm{d} C}$ Corresponding Author: Shengkun Sun, Department of Urology, Chinese PLA General Hospital, 28th, Fuxing Road, Beijing 100853, China. Email: saintkuns@gmail.com; Axiang Xu, Department of Urology, Chinese PLA General Hospital, 28th, Fuxing Road, Beijing 100853, China.

Email: axiangplagh@sina.com

doi: http://dx.doi.org/10.14740/wjnu221w

\section{Introduction}

Bladder cancer is the fourth most frequently diagnosed malignancy in males and the 12th most common malignancy in females [1]. Transurethral resection of bladder tumor (TURBT) remains the mainstay of diagnosis and initial treatment of bladder neoplasms. TURBT is performed conventionally with an electrocautery loop. The piecemeal resection specimens are inappropriate for tumor pathological evaluation because of poor anatomic orientation and evaluation of the pathological depth is not necessarily accurate. Also, tumor cells are scattered as the tumor is fragmented so that the possibility of implantation cannot be excluded. Several authors have reported on new technique for transurethral en bloc resection of bladder tumors, but little has been clarified regarding the quality of the obtained specimens as well as the obturator nerve reflex (ONR) during resection [2-5].

Rodriguez and colleagues demonstrated that the lateral wall harbors $46.8 \%$ of all bladder tumors [6]. ONR remains as a landmine during treating tumors nested in lateral bladder walls. Adductor muscle spasm is reported to occur in 55.3$100 \%$ of resections of lateral wall bladder tumors [6-8]. Bladder perforation, difficultly manageable bleeding, incomplete resection of tumor, and hazard of extravesical spread of tumor are major risks of this reflex [9]. Most reports favored obturator nerve block (ONB) as a safe procedure to prevent obturator jerks with various success rates [10-12]. Successful ONB performed in addition to spinal anesthesia in TURBT procedures for tumors involving the lateral wall can prolong time to recurrence and lengthen the disease-free survival [13]. However, there are also reports in the literature of complications related to ONB such as serious bleeding, seizures, disturbed vision, paralysis, nerve damage, and insufficient ONB $[14,15]$.

These inherent faults in TURBT imposed the need for new alternatives. The present study aimed to describe a simple yet reliable transurethral needle electrode resection of bladder tumor (TUNER-BT), which achieves en bloc resection while obviating obturator nerve stimulation.

\section{Methods}

The study included 46 patients planned for TURBT with nonmuscle-invasive bladder carcinoma located in the lateral blad- 


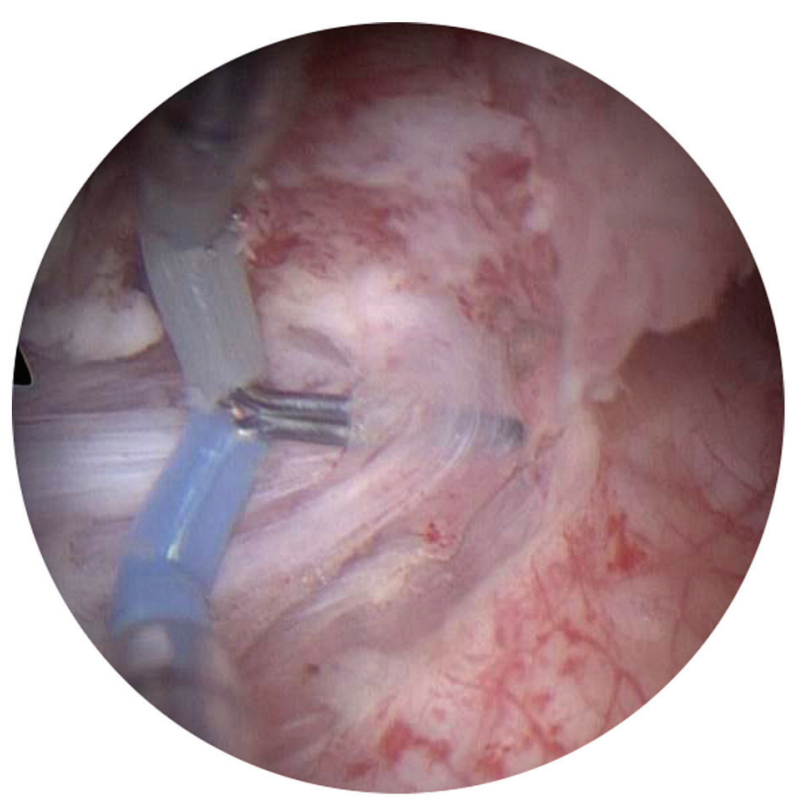

Figure 1. The needle tip was inserted into the muscle layer and the muscle bundles were stretched away from the lateral bladder wall.

der wall. Mean patient age was $61.5 \pm 13.2$ years, with 27 males and 19 females. Those who have tumors $>2.5 \mathrm{~cm}$ in diameter were excluded from analysis. All patients gave their written informed consent for the procedure. The study protocol was reviewed and approved by the Institutional Review Boards of Chinese PLA General Hospital. CT and cystoscopy were performed to diagnose bladder cancer and evaluate the preoperatively clinical stage. Intravenous urography was performed to exclude upper urinary tract tumors. The study was performed using a 26-F Karl Storz ${ }^{\circledR}$ continuous flow resectoscope (Karl Storz GmbH \& Co. KG, Tuttlingen, Germany) with $12^{\circ}$ lens. Bipolar resection was performed using the Plasma Kinetic Super Pulse Generator with a needle electrode (WA22355A). The power level was set as recommended by the manufacturer, delivering $160 \mathrm{~W}$ in cutting mode and $80 \mathrm{~W}$ in coagulation mode. Saline water was used as the operative rinse solution.

Senior consultant urologist with experience of TURBT performed all the resections under subdural anesthesia. No ONB was used. Patients were placed in the lithotomy position. The procedures started with a comprehensive cystoscopy, determining the presence, size, and location of all existing tumors. After electrocautery of the bladder mucosa $1 \mathrm{~cm}$ away from the tumor base by coagulation current, the needle tip was then inserted into the muscle layer and the muscle bundles were stretched away from the lateral bladder wall, followed by cutting with the electroresection current (Fig. 1). Then the tumor as well as its base was dissected bluntly with the needle. When there was a fibrous zone that could not be separated, it was incised. The intact tumor specimen as well as its basal layer was retrieved through the resectoscope sheath with the needle electrode and sent for pathological examination.

At the end of the procedure, a 20-F three-way Foley catheter was retained. Postoperative bladder irrigation was not performed unless hematuria occurred. Catheter will be re-

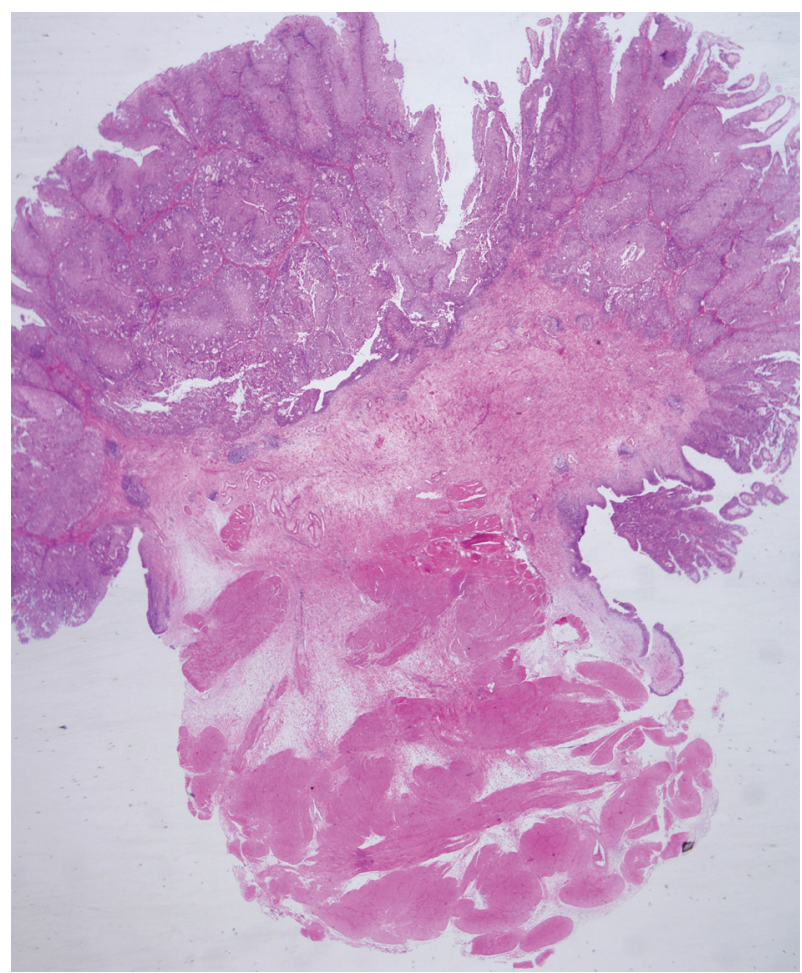

Figure 2. Resected specimen. Muscle layer is observed in beneath tumor base. $\mathrm{H} \& \mathrm{E}, \times 1.25$.

moved in 2 days after no gross hematuria. For every patient, parameters such as tumor number, size, location, tumor resection time, obturator jerks, perforation, and catheterization time were recorded. A pathologist evaluated the resected tissues for tumor grade and stage. The 2009 TNM classification was used for histopathologic examination [16]. Intravesical chemotherapy with $50 \mathrm{mg} / 50 \mathrm{~mL}$ epirubicin was applied weekly for 8 weeks after operation, followed by monthly maintenance to 12 months. All patients are followed with cystoscopy and ultrasonography at 3-month intervals. All complications within 90 days of surgery were recorded and graded according to an established five-grade Clavien classification [17].

\section{Results}

Since 2013 we have performed this technique on 56 lesions in 46 patients. Mean tumor diameter was $1.79 \pm 0.43 \mathrm{~cm}$ (range, $1.0-2.5 \mathrm{~cm})$. Tumors were multiple in $11(24 \%)$ patients and single in $35(76 \%)$ patients, with $24(42.9 \%)$ on the left lateral wall, and $32(57.1 \%)$ on the right lateral wall. Mean resection time was $10.8 \pm 4.8 \mathrm{~min}$ (range, 3 - $19 \mathrm{~min}$ ) for one tumor. No patients experienced ONR intraoperatively. Significant comorbidities such as bladder wall perforation, TUR syndrome, and severe postoperative bleeding did not occur. Mean catheterization time was $22.5 \pm 6.5 \mathrm{~h}$ (range, $8-36 \mathrm{~h}$ ) and only six patients required transient bladder irrigation after operation.

Complete resection was achieved in all patients. Histopathologic evaluation demonstrated that muscle lamina was 

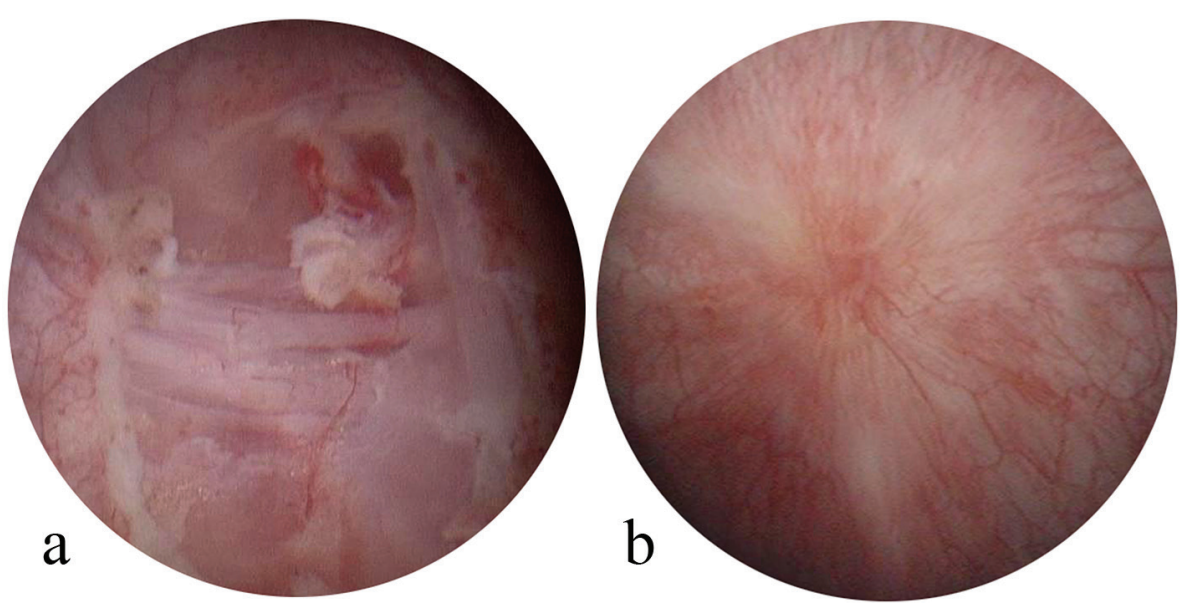

Figure 3. Scar healing of the tumor bed. (a) Postoperative wound bed. (b) Scar at 3-month follow-up.

identified in all cases. There was a clear margin of lamina propria and muscularis propria beneath the tumor base (Fig. 2). The pathologic examination identified 46 patients with transitional cell carcinoma (32.5\% pTa, 56.4\% pT1, and $11.1 \%$ pT2 cases in the series). Cystoscopy 3 months postoperatively showed scar healing of the operative wound (Fig. 3). Five cases experienced intravesical recurrence but none occurred at the resection site during a mean (range) follow-up of 13.6 (6 - 20) months. No patients had grade 2 or higher events according to the Clavien classification.

\section{Discussion}

TURBT is the current gold standard for treatment of non-muscle-invasive bladder cancer. Traditional TURBT, which is performed conventionally with an electrocautery loop, has been in practice for many years. A myriad of issues continue to plague TURBT such as incomplete resection, fragmented specimen invalid for precise pathological diagnosis, bladder perforation or bleeding due to ONR.

In the present study, a needle electrode replacing the conventional loop electrode during transurethral electroresection has greatly facilitated the operation. One advantage of TUNER$\mathrm{BT}$ is the excellent visibility during the procedure. Bleeding is most commonly encountered during TURBT. Conventional transurethral resection often starts from the tumor crown, and tumor vessels rooting from the base may be cut repeatedly at different levels. For a relatively large papilloma, the bleeding point may be concealed by the stalk of the tumor, making coagulation difficult and markedly decreasing the visualization. TUNER-BT starts from the bottom of the base and the feeding vessels were cauterized at an early stage, greatly reducing the subsequent bleeding during surgery. Most surgical procedures can be completed in a bloodless field. When there was bleeding at the wound surface, it was cauterized by the needle tip and coagulation of the bleeding point in healthy tissue can be accomplished easily. Therefore, in most patients no bladder irrigation was required postoperatively and a urinary catheter remained indwelling for no more than 1 day. In addition, the carbonization effect of needle electrode is minimal. Excellent visualization during TUNER-BT allows controlled resection, enabling the surgeon to differentiate the tumoral tissue from the healthy muscular fibers of the bladder wall.

Considering the fact that most patients were undergoing a primary surgery, the correct tumor staging was of the utmost importance. Traditional TURBT relies on in situ tumor fragmentation for tumor removal and specimen retrieval. This practice violates the basic oncological tenet of en bloc resection, promoting tumor cell dispersal and jeopardizing the pathological integrity of the specimen [18]. TURBT remains a surgery in which the end point is the presence of muscle but complete resection is not always achieved. The muscularis propria was absent in up to $40-51 \%$ of cases according to literature $[19,20]$. The absence of muscles was a significant predictor of clinical understaging and might mislead the therapeutic decision [21].

In the present series, TUNER-BT provides intact specimens containing detrusor muscle. We could reliably estimate the distance between the invasive front and the resection margin, which was helpful for precise pathological staging and follow-up treatment. Also, even large tumors are more likely to be resected en bloc with TUNER-BT, although removal of the resected tumors from the bladder is still expected to be difficult. This problem is intrinsic to transurethral surgery and is considered avoidable. The tumor can be cut into pieces that would pass through the urethra. However, in this series, we select tumors $<2.5 \mathrm{~cm}$ in diameter.

The risk of residual tumor after TURBT of Ta-T1 lesions ranges from $4 \%$ to $76 \%[19,22,23]$. To determine whether the tumor was removed completely, taking of biopsy specimens from the tumor bed should be routinely performed after resection. On the other hand, single small tumors are prone to vaporization during TURBT, especially in holmium laser TURBT, which results in insufficient specimens for postoperative pathologic diagnosis. So preoperative biopsies should be routinely performed to prevent the insufficient availability of postoperative specimens. With the present method, the presence of detrusor muscle in $100 \%$ cases would not only provide adequacy of the TURBT but also reduce the need for second-look TURBT. 
An additional biopsy from the tumor base is also not necessary. One of the most serious and frequently encountered complications during conventional TURBT is ONR, especially for tumors involving the lateral bladder wall [24]. ONR results in sudden spasm of the adductor muscles of the thigh, which may lead to a penal of severe complications such as partial or complete bladder perforation, bleeding, extravesical spread of tumor, and pelvic organ injury [25-28]. Spinal anesthesia does not prevent unintended stimulation of the obturator nerve when electroresection is performed. Although there are reports that occurrence of adductor contractions with bipolar TURBT is lower compared to monopolar TURBT [29], Venkatramani reported that bipolar TURBT was not superior to monopolar resection with respect to obturator jerk, bladder perforation and hemostasis [30]. The incidence of obturator jerk was $49.2 \%$ in the monopolar arm and $60 \%$ in the bipolar arm in their report. In our study, none of the patients with bladder tumors nested in the lateral wall experienced ONR or bladder perforation. The ONR privilege of TUNER-BT can be ascribed to the prolonged distance from the obturator nerve by drawing muscle bundles away from the bladder wall. Moreover, the slim tip of the needle electrode brought less stimulation of the obturator nerve compared with traditional electrocautery loop. Blunt stripping of the tumor base from lateral bladder wall without eletrostimulation also contributes to this ONR privilege.

In conclusion, TUNER-BT is a novel technique in transurethral resection with multiple advantages such as the absence of ONR, minimum intraoperative bleeding, and en bloc resection. The ONR privilege will free the urologists from the fear of obturator jerk. The en bloc specimen gives pathologists the opportunity for correct staging. Though our initial experience of TUNER-BT is limited, the results are encouraging and TUNER-BT seems to represent a promising endoscopic treatment for non-muscle-invasive bladder tumor. Since our median follow-up of 13.6 months was too short, longer follow-up periods and further prospective studies will be required to establish the long-term advantages and general viability of TUNER-BT as a therapeutic approach in bladder cancer.

\section{Financial Disclosures}

We declare that none of the authors have any conflict of interest, including specific financial interests or relationship and affiliations relevant to the subject matter or material discussed in the manuscript.

\section{References}

1. Siegel R, Ma J, Zou Z, Jemal A. Cancer statistics, 2014. CA Cancer J Clin. 2014;64(1):9-29.

2. Lodde M, Lusuardi L, Palermo S, Signorello D, Maier K, Hohenfellner R, Pycha A. En bloc transurethral resection of bladder tumors: use and limits. Urology. 2003;62(6):1089-1091.

3. Saito S. Transurethral en bloc resection of bladder tumors. J Urol. 2001;166(6):2148-2150.

4. Kawada T, Ebihara K, Suzuki T, Imai K, Yamanaka H. A new technique for transurethral resection of bladder tumors: rotational tumor resection using a new arched electrode. J Urol. 1997;157(6):2225-2226.

5. Ukai R, Hashimoto K, Iwasa T, Nakayama H. Transurethral resection in one piece (TURBO) is an accurate tool for pathological staging of bladder tumor. Int $\mathrm{J}$ Urol. 2010;17(8):708-714.

6. Garcia Rodriguez J, Jalon Monzon A, Gonzalez Alvarez RC, Ardura Laso C, Fernandez Gomez JM, Rodriguez Martinez JJ, Martinez Gomez FJ, et al. [An alternative technique to prevent obturator nerve stimulation during lateral bladder tumours transurethral resection]. Actas Urol Esp. 2005;29(5):445-447.

7. Deliveliotis C, Alexopoulou K, Picramenos D, Econorna$\cos$ G, Goulandris N, Kostakopoulos A. The contribution of the obturator nerve block in the transurethral resection of bladder tumors. Acta Urol Belg. 1995;63(3):51-54.

8. Tatlisen A, Sofikerim M. Obturator nerve block and transurethral surgery for bladder cancer. Minerva Urol Nefrol. 2007;59(2):137-141.

9. Thallaj A, Rabah D. Efficacy of ultrasound-guided obturator nerve block in transurethral surgery. Saudi J Anaesth. 2011;5(1):42-44.

10. Khorrami M, Hadi M, Javid A, Izadpahani MH, Mohammadi Sichani M, Zargham M, Alizadeh F. A comparison between blind and nerve stimulation guided obturator nerve block in transurethral resection of bladder tumor. $\mathrm{J}$ Endourol. 2012;26(10):1319-1322.

11. Jo YY, Choi E, Kil HK. Comparison of the success rate of inguinal approach with classical pubic approach for obturator nerve block in patients undergoing TURB. Korean J Anesthesiol. 2011;61(2):143-147.

12. Kakinohana M, Taira Y, Saitoh T, Hasegawa A, Gakiya M, Sugahara K. Interadductor approach to obturator nerve block for transurethral resection procedure: comparison with traditional approach. J Anesth. 2002;16(2):123-126.

13. Tekgul ZT, Divrik RT, Turan M, Konyalioglu E, Simsek E, Gonullu M. Impact of obturator nerve block on the short-term recurrence of superficial bladder tumors on the lateral wall. Urol J. 2014;11(1):1248-1252.

14. Takeuchi M, Hirabayashi Y, Hotta K, Inoue S, Seo N. [Ropivacaine-induced grand mal convulsion after obturator nerve block]. Masui. 2005;54(11):1309-1312.

15. Akata T, Murakami J, Yoshinaga A. Life-threatening haemorrhage following obturator artery injury during transurethral bladder surgery: a sequel of an unsuccessful obturator nerve block. Acta Anaesthesiol Scand. 1999;43(7):784-788.

16. Sobin LH, Gospodariwicz MK, Wittekind C. TNM classification of malignant tumours. UICC International Union Against Cancer. 7th ed. New York, NY: Wiley-Blackwell, 2009; pp.262-265.

17. Dindo D, Demartines N, Clavien PA. Classification of surgical complications: a new proposal with evaluation in a cohort of 6336 patients and results of a survey. Ann Surg. 2004;240(2):205-213.

18. Maurice MJ, Ponsky LE. En bloc transurethral resection of bladder lesions: a trick to retrieve specimens up to 4.5 cm. BJU Int. 2013;111(3):E15-16. 
19. Herr HW. The value of a second transurethral resection in evaluating patients with bladder tumors. J Urol. 1999;162(1):74-76.

20. Maruniak NA, Takezawa K, Murphy WM. Accurate pathological staging of urothelial neoplasms requires better cystoscopic sampling. J Urol. 2002;167(6):2404-2407.

21. Dutta SC, Smith JA, Jr., Shappell SB, Coffey CS, Chang SS, Cookson MS. Clinical under staging of high risk nonmuscle invasive urothelial carcinoma treated with radical cystectomy. J Urol. 2001;166(2):490-493.

22. Babjuk M, Oosterlinck W, Sylvester R, Kaasinen E, Bohle A, Palou-Redorta J, Roupret M. EAU guidelines on non-muscle-invasive urothelial carcinoma of the bladder, the 2011 update. Eur Urol. 2011;59(6):997-1008.

23. Brausi M, Collette L, Kurth K, van der Meijden AP, Oosterlinck W, Witjes JA, Newling D, et al. Variability in the recurrence rate at first follow-up cystoscopy after TUR in stage Ta T1 transitional cell carcinoma of the bladder: a combined analysis of seven EORTC studies. Eur Urol. 2002;41(5):523-531.

24. Dorotta I, Basali A, Ritchey M, O'Hara JF, Jr., Sprung J. Transurethral resection syndrome after bladder perforation. Anesth Analg. 2003;97(5):1536-1538.

25. Puppo P, Bertolotto F, Introini C, Germinale F, Timossi L,
Naselli A. Bipolar transurethral resection in saline (TURis): outcome and complication rates after the first 1000 cases. J Endourol. 2009;23(7):1145-1149.

26. Traxer O, Pasqui F, Gattegno B, Pearle MS. Technique and complications of transurethral surgery for bladder tumours. BJU Int. 2004;94(4):492-496.

27. Geavlete B, Multescu R, Georgescu D, Stanescu F, Jecu M, Geavlete P. Narrow band imaging cystoscopy and bipolar plasma vaporization for large nonmuscleinvasive bladder tumors--results of a prospective, randomized comparison to the standard approach. Urology. 2012;79(4):846-851.

28. Cesur M, Erdem AF, Alici HA, Yapanoglu T, Yuksek MS, Aksoy Y. The role of succinylcholine in the prevention of the obturator nerve reflex during transurethral resection of bladder tumors. Saudi Med J. 2008;29(5):668-671.

29. Gupta NP, Saini AK, Dogra PN, Seth A, Kumar R. Bipolar energy for transurethral resection of bladder tumours at low-power settings: initial experience. BJU Int. 2011;108(4):553-556.

30. Venkatramani V, Panda A, Manojkumar R, Kekre NS. Monopolar versus bipolar transurethral resection of bladder tumors: a single center, parallel arm, randomized, controlled trial. J Urol. 2014;191(6):1703-1707. 